\title{
Shear-induced chaos in nonlinear Maxwell-model fluids
}

\author{
Chris Goddard* and Ortwin Hess ${ }^{\dagger}$ \\ Advanced Technology Institute, School of Electronics and Physical Sciences, University of Surrey, Guildford, GU2 7XH, United Kingdom \\ Alexander G. Balanov \\ Department of Physics, Loughborough University, Loughborough, LE11 3TU, United Kingdom \\ Siegfried Hess \\ Institut für Theoretische Physik, Technische Universität Berlin, Hardenbergstrasse 36, D-10623 Berlin, Germany
}

(Received 3 July 2007; published 29 February 2008)

\begin{abstract}
A generalized model for the behavior of the stress tensor in non-Newtonian fluids is investigated for spatially homogeneous plane Couette flow, showing a variety of nonlinear responses and deterministic chaos. Mapping of chaotic solutions is achieved through the largest Lyapunov exponent for the two main parameters: The shear rate and the temperature and/or density. Bifurcation diagrams and stability analysis are used to reveal some of the rich dynamics that can be found. Suggested mechanisms for stability loss in these complex fluids include Hopf, saddle-node, and period-doubling bifurcations.

DOI: 10.1103/PhysRevE.77.026311

PACS number(s): 47.20.Ky, 47.20.Gv, 47.27.ed, 47.50.-d
\end{abstract}

\section{INTRODUCTION}

Non-Newtonian fluid behavior, which is characterized by a nonlinear viscosity dependence on the strain, can be observed in many complex fluids, for example, polymers, dense colloidal dispersions, surfactant solutions, and micellar solutions. In addition to shear-thinning and shear-thickening behavior, a dynamic or even chaotic response can be found in some fluids subjected to a steady shear flow. Phenomenologically closely related is the stick-slip behavior associated with plastic flow occurring in dry friction processes.

A nonlinear generalization of the time honored Maxwell fluid provides a generic model for the treatment of both shear-thinning and shear-thickening behavior [1]. Recently, it has been demonstrated that the nonlinear Maxwell model also yields periodic and chaotic solutions for specific ranges of the relevant model parameters [2]. The flow curve, i.e., the dependence of the viscosity or of the shear stress on the shear rate can be invoked to correlate the model with a physical system. An example to be specified below is a shear-thickening micellar solution $[3,4]$

In recent years there has been an increase of interest in the behavior of complex fluids under flow. This is shown by the amount of research being undertaken by people in the community. Cates and co-workers [5-7] study simple constitutive equations for shear-thickening materials and show instabilities and chaos under shear flow. Das et al. [8] focus on routes to spatiotemporal chaos in sheared nematogenic fluids, an area also investigated by Fielding and Olmsted [9] using simple models. Dasan et al. [10] use the tools of nonlinear dynamics to study stress fluctuations in sheared Stokesian suspensions.

Most of this work was inspired by the study of models for the dynamics of liquid crystals based on the alignment tensor

\footnotetext{
*c.goddard@surrey.ac.uk

†o.hess@ surrey.ac.uk
}

theory [11-13] (which, in limiting cases, reduces to the Leslie-Ericksen theory) and Doi-Hess kinetic theory of nematic polymers [14-16]. The orientation of the director and degree of alignment allowed for calculations of rheological properties, which is the step taken by Rienäcker et al. [17-19] to eventually show chaotic rheological behaviors. A close similarity in the behavior of the stress and the alignment tensor is found in many fluids and referred to as the stress-optical law [20], which motivates our choice of equations.

Although it is known that such systems are able to demonstrate a variety of dynamical regimes, including chaos, the instabilities and typical transitions between such regimes are weakly studied. In this paper we systematically study dynamical mechanisms for the evolution of different regimes and reveal typical instabilities leading to the onset of chaos. More specifically, a solution phase diagram based on the largest Lyapunov exponent is presented which indicates the kind of solutions which can occur in distinct ranges of the model parameters. The transition from periodic to chaotic solutions is investigated. Period doubling and also undoubling scenarios are found for certain parameter ranges. In other regimes, Shilnikov type limit cycle solutions and an intermittency route to chaos are found. These findings are compared to parametric bifurcation diagrams which allow for a better classification of the bifurcation types and reveal in more detail the dynamics behind the equations.

The present analysis is restricted to the study of a simple shear flow, i.e., a plane Couette geometry with an imposed spatially constant shear rate, yet the dynamics is already rather complex. We consider such an analysis as compulsory before the full hydrodynamics with spatially inhomogeneous shear rates as encountered in particular experiments is investigated. At least on a small length scale, the relation between the local stress tensor and a local shear rate as studied here also exists for more complex flows, and knowing the local features can be crucial to understanding the global behavior. 


\section{MODEL}

We study a phenomenological model for the evolution of the stress tensor in complex fluids for simple plane Couette flow to investigate the nonlinear processes at work. The stress tensor is decomposed into a contribution associated with the internal structure for which the nonlinear Maxwell model equations are formulated and a contribution linked with a second Newtonian viscosity $\eta_{\infty}$ reached at high shear rates,

$$
\overline{\sigma_{\mu \nu}}=\sqrt{2} G_{\text {ref }} \pi_{\mu \nu}+2 \eta_{\infty} \gamma_{\mu \nu}
$$

Here $\overline{\sigma_{\mu \nu}}$ is the symmetric traceless part of the stress tensor, $G_{\text {ref }}$ is a reference value for the shear modulus such that $\pi_{\mu \nu}$ is a dimensionless friction stress tensor, and $\gamma_{\mu \nu}$ is the strain (deformation) rate. Greek subscripts indicate Cartesian components and the summation convention is used. The friction stress tensor $\pi_{\mu \nu}$ is assumed to obey the generalized Maxwell model equation [1],

$$
\begin{aligned}
& \frac{\partial}{\partial t} \pi_{\mu \nu}-2 \bar{\epsilon}_{\mu \lambda \kappa} \omega_{\lambda} \bar{\pi}_{\kappa \nu}-2 \kappa \bar{\gamma}_{\mu \lambda} \bar{\pi}_{\lambda \nu}+\tau_{0}^{-1}\left(\Phi_{\mu \nu}-\ell_{0}^{2} \Delta \pi_{\mu \nu}\right) \\
& \quad=\sqrt{2} \gamma_{\mu \nu},
\end{aligned}
$$

where $\omega_{\lambda}$ is the vorticity, $\Phi_{\mu \nu}$ is the derivative of a potential function, $\tau_{0}$ is a relaxation time, $\ell_{0}$ is a characteristic length, and $\kappa$ is a model parameter. The spatial aspect of this model is not considered in these investigations.

The potential function corresponds to an expansion of $\Phi$ up to terms of fourth order in $\pi_{\mu \nu}$, using the second and third order invariants $I_{2}$ and $I_{3}$. The ordinary Maxwell model equation is linear in the stress tensor, and the nonlinear generalization was invented to treat shear thickening and shear thinning behavior [1]. The potential term reads (after a rescaling of variables)

$$
\Phi_{\mu \nu}=A^{*} \pi_{\mu \nu}-3 \sqrt{6} \bar{\pi}_{\mu \lambda} \pi_{\lambda \nu}+2 \pi_{\mu \nu} \pi_{\lambda \kappa} \pi_{\lambda \kappa} .
$$

After considerations for plane Couette flow (externally imposed field) the system can be expressed through basis tensors as a system of five coupled homogeneous differential equations for the components of the stress tensor. They are presented here in their final form, although for complete derivations and fundamental properties refer to the relevant publications $[2,1]$,

$$
\begin{aligned}
\dot{\pi}_{0}= & -\left[A^{*}-3 \pi_{0}+2(\underline{\underline{\pi}})^{2}\right] \pi_{0}-3\left(\pi_{1}^{2}+\pi_{2}^{2}\right) \\
& +\frac{3}{2}\left(\pi_{3}^{2}+\pi_{4}^{2}\right)-\widetilde{\kappa} \Gamma \pi_{2}, \\
\dot{\pi}_{1}=-\left[A^{*}\right. & \left.+6 \pi_{0}+2(\underline{\underline{\pi}})^{2}\right] \pi_{1}+\frac{3}{2} \sqrt{3}\left(\pi_{3}^{2}-\pi_{4}^{2}\right)+\Gamma \pi_{2}, \\
\dot{\pi}_{2}= & -\left[A^{*}+6 \pi_{0}+2(\underline{\underline{\pi}})^{2}\right] \pi_{2}+3 \sqrt{3} \pi_{3} \pi_{4} \\
& +\pi_{c}^{-1} \Gamma-\Gamma\left(\widetilde{\kappa} \pi_{0}+\pi_{1}\right),
\end{aligned}
$$

$$
\begin{aligned}
\dot{\pi}_{3}= & -\left[A^{*}-3 \pi_{0}+2\left(\underline{\underline{\pi}}^{2}\right] \pi_{3}+3 \sqrt{3}\left(\pi_{1} \pi_{3}+\pi_{2} \pi_{4}\right)\right. \\
& +\frac{1}{2} \Gamma \pi_{4}(1+\sqrt{3} \widetilde{\kappa}), \\
\dot{\pi}_{4}= & -\left[A^{*}-3 \pi_{0}+2(\underline{\underline{\pi}})^{2}\right] \pi_{4}+3 \sqrt{3}\left(\pi_{2} \pi_{3}-\pi_{1} \pi_{4}\right) \\
& -\frac{1}{2} \Gamma \pi_{3}(1-\sqrt{3} \widetilde{\kappa}) .
\end{aligned}
$$

Here the dot denotes differentiation with respect to a scaled time variable $t$. The abbreviation

$$
(\underline{\underline{\pi}})^{2}=\pi_{2}^{2}+\pi_{1}^{2}+\pi_{0}^{2}+\pi_{3}^{2}+\pi_{4}^{2}
$$

is also used. Due to the scaling chosen, the relaxation time is characterized by the single parameter $A^{*}$. For $A^{*} \gg 1$, the nonlinear terms are less important and the ordinary Maxwell model behavior is approached. With $\kappa \neq 0$, this corresponds to the Johnson-Segalman model $[21,22]$. The yield stress at $A^{*}=1$ is denoted by $\pi_{c}$. Furthermore, $\widetilde{\kappa}=\kappa / \sqrt{3}$.

In the following investigations we have chosen to fix the values of $\pi_{c}=1$ and $\widetilde{\kappa}=0$ for simplicity. Based on the analysis of the related liquid crystal problem [19] for the plane Couette flow we expect that $\kappa \neq 0$ will give rise to quantitative changes but will not affect in a qualitative way the occurrence of the different types of the dynamical behavior.

The model is formulated such that $\pi_{0}, \pi_{1}$, and $\pi_{2}$ describe the main dynamics of the system in time through the shear stress and the first and second normal stress differences, while $\pi_{3}$ and $\pi_{4}$ provide symmetry breaking components for the plane Couette flow.

The dimensionless shear stress is expressed as

$$
\sigma=H \Gamma=\pi_{c} \pi_{2}+H_{\infty} \Gamma,
$$

where $\pi_{c}$ and $H_{\infty}$ are two model parameters set as constant in this investigation. $H_{\infty}$ is linked to the second Newtonian viscosity reached at high shear rates. The dimensionless first and second normal stress differences can also be computed from

$$
\begin{gathered}
N_{1}=\frac{\sigma_{x x}-\sigma_{y y}}{G}=2 \pi_{c} \pi_{1}, \\
N_{2}=\frac{\sigma_{y y}-\sigma_{z z}}{G}=-\pi_{c}\left(\pi_{1}+\sqrt{3} \pi_{0}\right) .
\end{gathered}
$$

The two control parameters chosen for investigation are $\Gamma$, which is a dimensionless shear rate for the plane Couette flow $\left(\Gamma=\partial v_{x} / \partial y\right)$, and $A^{*}$, which controls the strength of the linear terms in the potential function and depends strongly on the temperature of the system or the number density of the dispersion [2].

For realistic physical systems, the range of values available for investigation are $\Gamma=0 \rightarrow 5$ and here we focus on $A^{*}=0 \rightarrow 0.5$. As shown below, in these ranges of the parameters the system demonstrates a variety of interesting nonlinear phenomena including chaotic behavior.

For comparison with physical systems we notice that a shear-induced transition with an upward jump of the shear 


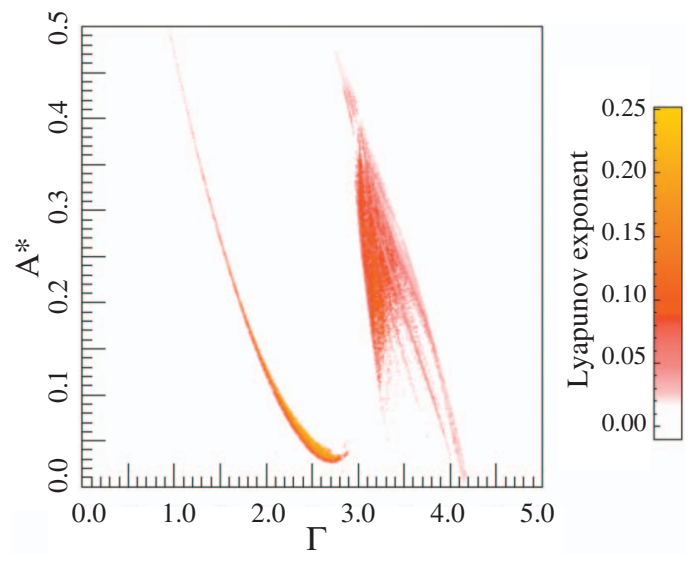

FIG. 1. (Color) Lyapunov exponent results for combinations of parameters $\Gamma$ and $A^{*}$. The higher the value, the more chaotic the solution. White areas are nonchaotic, either limit cycle or steady state; see Fig. 2. There are two distinct islands of chaos, which have very different dynamics.

stress for a shear rate controlled flow as it is observed in shear-thickening micellar solutions occurs for $A^{*}<1.5$. The experimental flow curve presented in Ref. [4] is matched by our model curve for $A^{*} \approx 1.2$ (with $\kappa=0$ and $\pi_{c}=1$ ). The reference values for the shear stress and the shear rate are $\approx 10 \mathrm{~Pa}$ and $100 \mathrm{~s}^{-1}$. It should be possible to reach the interesting regime of $A^{*}<0.5$ by lowering the temperature or by increasing the density. On the other hand, the plastic flow as encountered in solid friction shows many dynamic features [36-38] which are found for the present model in the range of $A^{*}$ investigated here.

\section{DYNAMICAL REGIMES}

First, we identify the regimes of chaotic dynamics of the system (4) by calculating the largest Lyapunov exponent in dependence of control parameters $\Gamma$ and $A^{*}$. If the largest exponent is zero or less, this signifies a regular oscillation in the system; a positive exponent however indicates a chaotic behavior.

The largest Lyapunov exponents calculated for different solutions are displayed in Fig. 1 through an intensity plot. The initial starting values for the components $\pi_{0}, \ldots, \pi_{4}$ are chosen to be small but nonzero, namely \pm 0.01 .

The figure shows two distinct "chaotic islands" which are separated by areas of regular dynamics. Generally, the parameter plane $\Gamma-A^{*}$ can be divided into four characteristic regions denoted in Fig. 2 by different colors, where the system demonstrates, from an experimental point of view, different dynamical regimes.

Here, region $S$ corresponds to steady state solutions, $P$ is an area where the attractor of the system is a single stable periodic orbit, and $M$ is a region of multistabilty, where several attractors, one of which might be a chaotic one, can coexist for the same parameters' values. The region $C$ contains the largest chaotic island shown in Fig. 1. This is an area where chaos is most likely to appear.

The steady state solutions from the model correspond experimentally to smooth equilibrium flows. The stresses set up

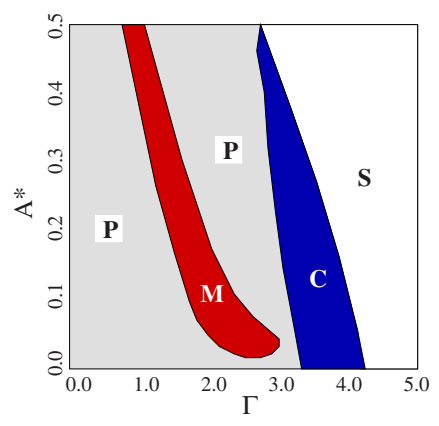

FIG. 2. (Color online) Solution phase diagram of the states of the system, taken from Fig. 1 . Solutions in region $P$ are stable periodic orbits, in $M$ there multiple orbits, one bifurcates to chaos and the other is stable, region $C$ contains chaotic solutions, and region $S$ are steady state.

in the fluid become constant (after some initial transient period) and represent normal laminar Couette flow responses for the imposed external flow field.

Periodic solutions in experiments can be brought about by many different causes. In the case of liquid crystals a tumbling or wagging motion of the director will produce such a response in the rheological properties [23]. For a lyotropic lamellar phase, the sample can oscillate between two distinct microstructural states [24]; for Micelles, temporal oscillations can be observed due to coupling between the microstructure and flow field [25].

Chaotic fluctuations of the shear stress are also observed experimentally, and caused by many of the same reasons as periodic flow, except that the mechanism at work results in irregular chaotic oscillations. For example, with a lyotropic lamellar phase, simple rheological chaos is observed when there is a competition between ordered and disordered states, driven by the stress [26]. Surfactant solutions show chaotic dynamics due to stick-slip between shear induced structures and the coexisting dilute phases [27].

Viscoelasticity is also a key property of this model and is a cause for the nonlinear behavior seen in the flow solutions from our simulations due to the stress history dependence. Experimentally, viscoelastic polymer solutions have been seen to display chaotic effects at low Reynolds numbers due to elastic turbulence, driven by elastic stresses causing instabilities [28].

\section{BIFURCATION ANALYSIS}

In order to reveal instabilities that induce transitions between the regions in Fig. 2 and that lead to the appearance of chaotic behavior we undertook a bifurcation analysis of the model (4). The results of this analysis for the most important regimes are summarized in Fig. 3, where different bifurcation lines are indicated by different colors. The bifurcation diagram was revealed using numerical branch continuation methods [29] and stability analysis.

A comparison of Fig. 2 and Fig. 3 shows that the boundary of the regions in Fig. 2 are in good agreement with certain bifurcation lines in Fig. 3. In particular, we can conclude 


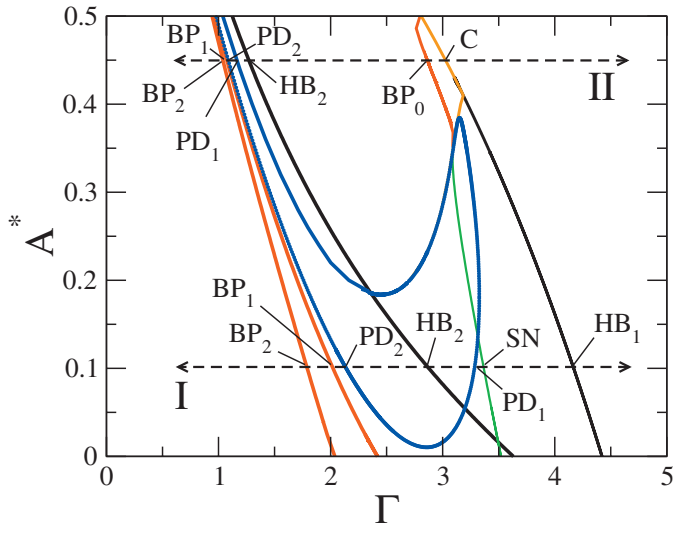

FIG. 3. (Color) Bifurcation diagram in the parameters' plane $\Gamma$ and $A^{*}$. Hopf bifurcation (black), saddle-node bifurcation (green), line of branching (red), attractor crisis (orange), and period doubling bifurcation (blue).

that the boundary between the regions $P$ and $M$ in Fig. 2 involve lines of Andronov-Hopf (in Fig. 3, a black line containing the points $\mathrm{HB}_{2}$ ), period-doubling bifurcations (blue curve in Fig. 3), and a branching line (containing the points $\mathrm{BP}_{2}$ ). The boundaries of the region $C$ also contain several bifurcation lines. The transition between the regions $S$ and $C$ is associated either with Andronov-Hopf bifurcation (see a black line containing the point $\mathrm{HB}_{1}$ in Fig. 3) or with a line of crisis (orange line containing the point $C$ ), whereas the transition between $P$ and $C$ involves a saddle-node (fold) bifurcation of limit cycles (green line) and a branching line (red line containing the point $\mathrm{BP}_{0}$ ).

Figure 4 illustrates the main bifurcations that the system undergoes with variation of the parameter $\Gamma$ along two characteristic routes indicated in Fig. 3 by arrows. Figure 4(a) shows a typical one-parametrical bifurcation diagram for relatively small $A^{*}=0.1$ (route $I$ in Fig. 3 ). The vertical axis is the value of $\pi_{2}$ in the Poincaré section. For fixed points, this is just their coordinate $\pi_{2}$; for limit cycles, this is a maximal $\pi_{2}$ value on the period. Notice that a negative value of $\pi_{2}$ found at high shear rates does not imply a negative shear stress since the total stress contains an additional contribution involving the second Newtonian viscosity; cf. Eq. (1). For a discussion of the entropy production, see [2].

The following abbreviations are used in Fig. 4: HBAndronov-Hopf bifurcation; PD-period-doubling bifurcation; $\mathrm{SN}$ - saddle-node (fold) bifurcation for periodic orbits; $\mathrm{BP}$ - branch point.

One can distinguish three main families of periodic orbits which are involved in dynamics of the model (4) and give rise to all stable oscillatory solutions. In the figure the branches of the above orbits are indicated by numbers $1-3$. As the value of $\Gamma$ decreases, the family of cycles 1 appears through Hopf bifurcation $\left(\mathrm{HB}_{1}\right)$. The initial limit cycle of this family loses its stability after it has undergone a perioddoubling bifurcation at the point $\mathrm{PD}_{1}(\Gamma \approx 3.3)$, which leads to the appearance of a stable period-doubled cycle. Further decrease of $\Gamma$ produces a cascade of period-doubling bifurcations, which eventually leads to the birth of chaos. Note, to prevent overloading of the diagram only the main cycles of each family are shown in the figure. At $\Gamma \approx 2.15$, the main
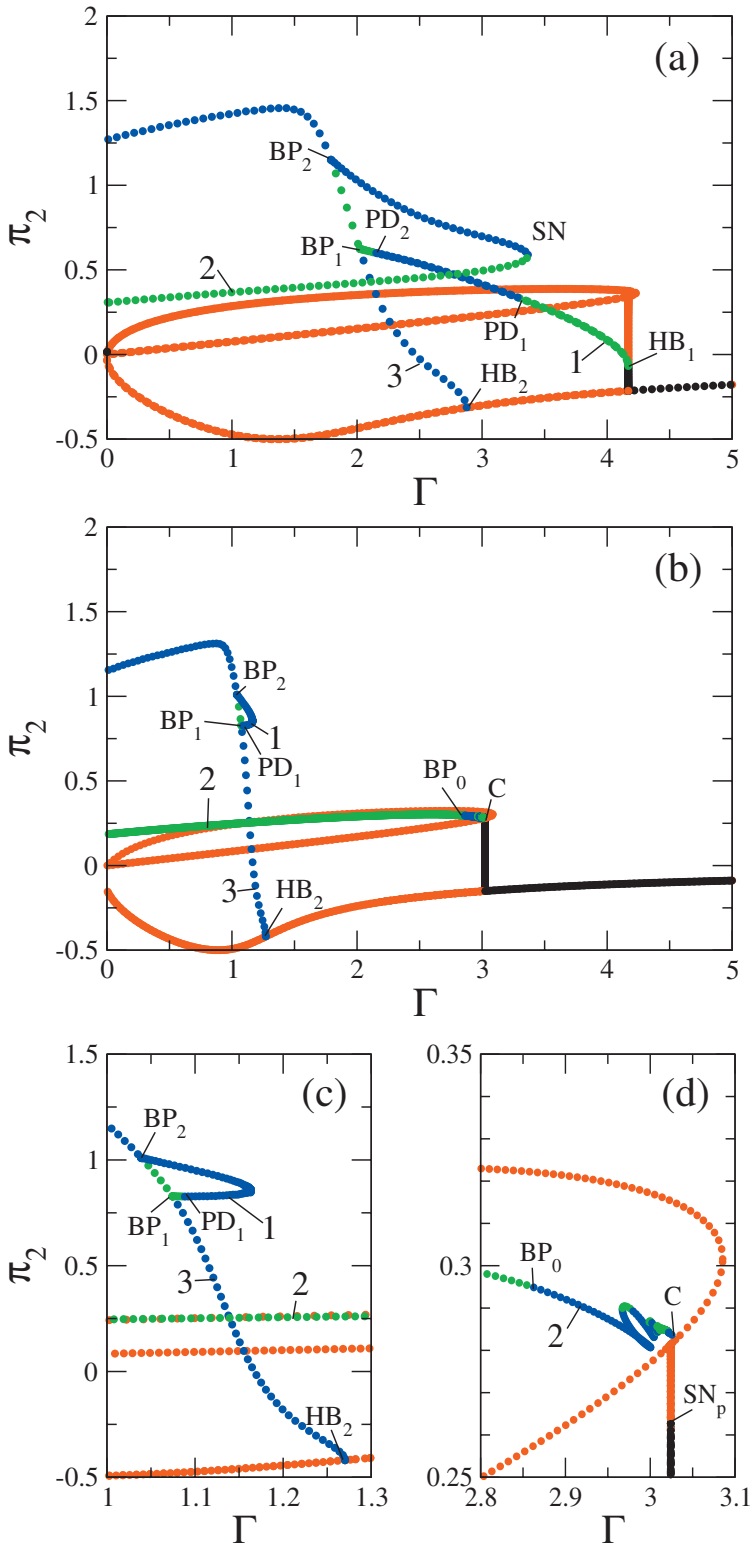

FIG. 4. (Color) One-parametric bifurcation diagrams: (a) $A^{*}$ $=0.1$; (b) $A^{*}=0.45$; (c) and (d) are zoomed parts of (b). The vertical axis is coordinate $\pi_{2}$ for fixed points or maximal value of $\pi_{2}$ for periodic orbits. Stable fixed point (black), unstable fixed point (red), stable periodic orbit (green), and unstable periodic orbit (blue).

cycle of the family 1 again becomes stable as the result of an inverse period-doubling bifurcation (point $\mathrm{PD}_{2}$ ). At $\Gamma \approx 2$ $\left(\mathrm{BP}_{1}\right)$ it merges with a cycle of family 3 . As $\Gamma$ decreases, the family 2 appears via saddle-node bifurcation for periodic orbits (SN). This bifurcation gives birth to a pair of cycles, one of which is stable (belongs to family 2), and one which is unstable (period-doubled cycle of the branch of family 3 ). Remarkably, the stable limit does not change its stability with further decreasing down to $\Gamma=0$, and, in fact, its existence determines the region $P$ in Fig. 2 for small values of $\Gamma$. The initial cycle of family 3 is born unstable from an unstable fixed point at $\Gamma \approx 2.8$ via Andronov-Hopf bifurcation $\left(\mathrm{HB}_{2}\right)$. After merging with family 1 it becomes stable at $\mathrm{BP}_{1}$ 
and then again loses its stability at $\mathrm{BP}_{2}$ as the result of subcritical period-doubling bifurcation.

The presence of three different families of periodic orbits predefines conditions for multistabilty phenomena, which dominates in the region $M$ in Fig. 2. For instance, on the interval $\Gamma \in(1.82,2.04)$ multistability manifests itself in the coexistence of stable limit cycles belonging to the families 2 and 3 , whereas on $\Gamma \in[2.04,2.14)$ it results from coexistence of the attractors of the families 1 and 2.

The $\pi_{3}$ and $\pi_{4}$ components change the type of solution depending on whether they are zero or nonzero. Nonzero components produce "kayaking" responses [18]. For the main cycles involved in the bifurcation diagram, only branch 3 has zero $\pi_{3}$ and $\pi_{4}$, while the main cycles of branches 1 and 2 have nonzero $\pi_{3}$ and $\pi_{4}$.

A different bifurcation scenario is realized for larger $A^{*}$, when $\Gamma$ changes along the route II in Fig. 3. In Figs. 4(b)-4(d) typical bifurcation transitions of the main cycles of families $1-3$ are illustrated for $A^{*}=0.45$. One can see that as before family 1 joins family 3 at a branching point $\mathrm{BP}_{1}$ [Fig. 4(c)]. However, in contrast to route I, family 1 does not involve anymore Andronov-Hopf bifurcations of a fixed point [see point $\mathrm{HB}_{1}$ in Fig. 4(a)]. Instead, the stability of the fixed point changes via saddle-node bifurcation $\mathrm{SN}_{p}$ [see Fig. 4(d)]. The evolution of the limit cycles of family 2 also changes. If along route I it loses stability as the result of saddle-node bifurcation merging with the unstable perioddouble cycle of family 3 , now it exists for larger values of $\Gamma$ until it collides with an unstable fixed point [Fig. 4(d)]. This collision is illustrated in Fig. 5, where the involved limit cycle and fixed point are shown for three values of the parameter $\Gamma$. As $\Gamma$ decreases, the limit cycle changes its shape and approaches an unstable point to collide with the latter at $\Gamma \approx 3.02$.

Note, comparing with route I, the evolution of family 2 becomes more complex. As it is seen from Fig. 4(d), with an increase of $\Gamma$, the main cycle of family 2 undergoes a number of bifurcations altering its stability and producing a few new branches of solutions. Hence, increasing $\Gamma$ for large values of $A^{*}$ can lead to complex dynamical behavior of the system, including chaos.

\section{MECHANISMS OF CHAOS}

Next we consider in more detail the scenario of developing chaos. To do this we use one-parametrical bifurcation analysis to scan the solutions of the system (4) across region $C$ in Fig. 2.

In Fig. 6 a one parametrical bifurcation diagram is presented which illustrates a Poincaré section $\pi_{2}$ of stable solution changes with varying $\Gamma$ and fixed $A^{*}=0.25$. As the parameter $\Gamma$ is increased up to $\Gamma=3.4446$, the system demonstrates a period-doubling transition to chaos. However, further increase of $\Gamma$ produces inverse sequences of bifurcations, where the period of a stable limit cycle is halved with each bifurcation. We found the period-doubling scenario for the onset of chaos quite general for the system (4). This type of chaos can be achieved for all main branches of solutions $1-3$; however, typically it is realized for a very

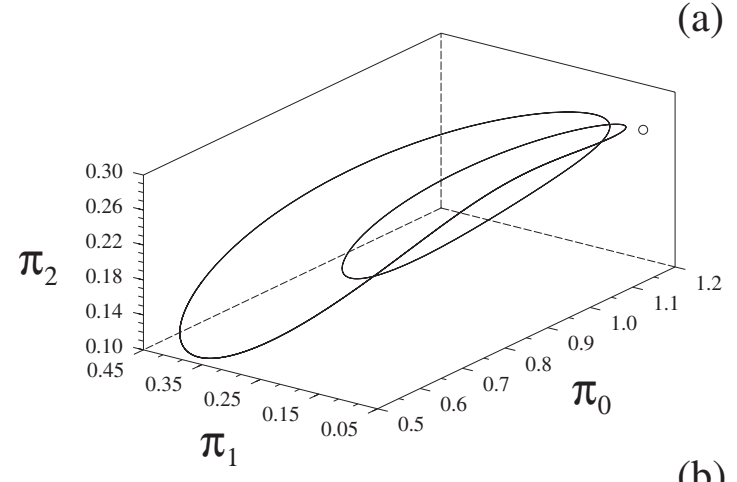

(a)

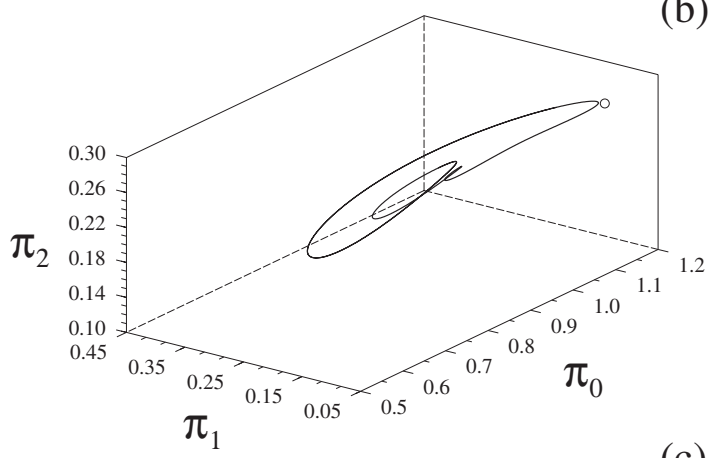

(c)

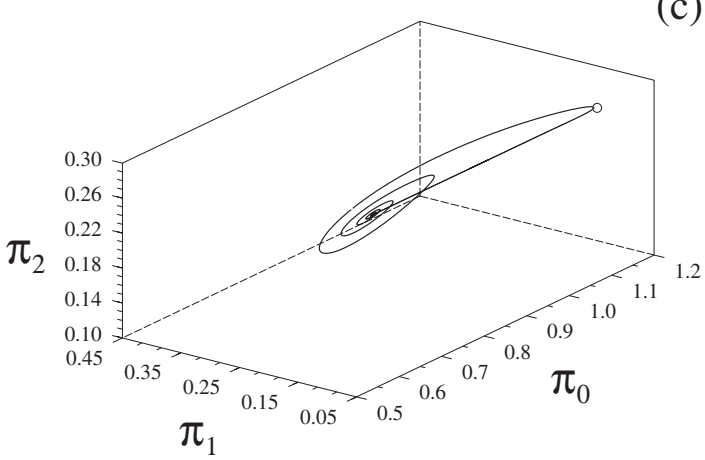

FIG. 5. Stable limit cycle of family 2 and unstable fixed point for $A^{*}=0.45$ and (a) $\Gamma=2.9$, (b) $\Gamma=3.01$, and (c) $\Gamma=3.02358$.

tiny range of parameters, which would be difficult to achieve in real experiments due to instrumental limitations.

Another type of chaos, which is different from the perioddoubling one, is illustrated in Fig. 7, where the time realization and a projection of the phase portrait are plotted for

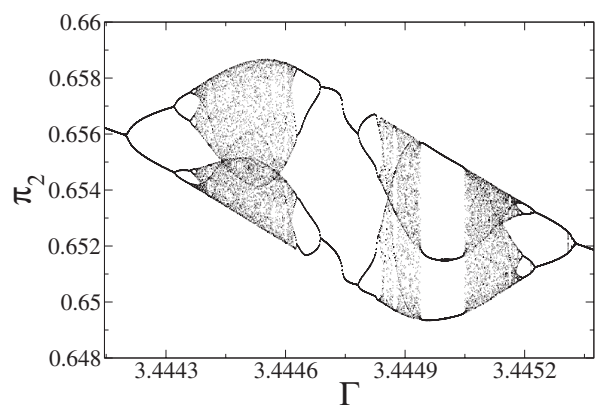

FIG. 6. Example of the type of period doubling transition to chaos in the region $C$ (see Fig. 2) for $A^{*}=0.25$. The Poincaré section used in the diagram is $\pi_{2}$. 


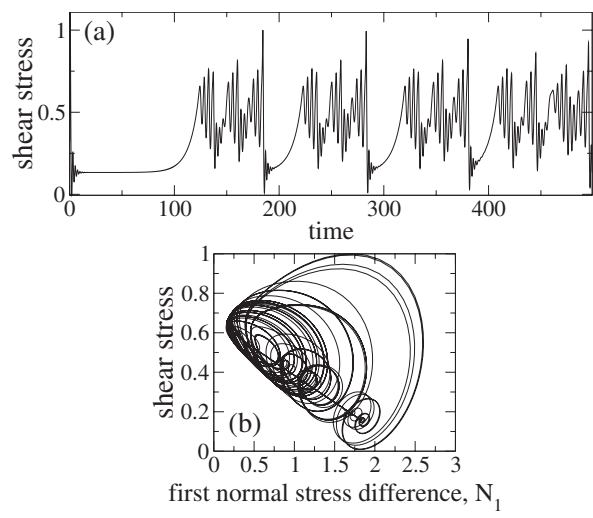

FIG. 7. (a) Example of the typical behavior found in region $C$ of the phase diagram. Shows a time series solution of the shear stress with intermittent relaxation oscillations. Parameters $A^{*}=0.3$ and $\Gamma$ $=3.3$ chosen. (b) Phase portrait projection has also been plotted.

$A^{*}=0.3$ and $\Gamma=3.3$. Here we see the system exhibiting interesting relaxation oscillations, where the stress slowly builds up and then reaches a point where, in this example, chaotic oscillations begin. Then at seemingly random points the solution essentially "drops" out of this normal nonlinear orbit (at $t=190,280,460, \ldots$ ), before slowly returning once again.

It is possible through further selection of model parameters to have the system stabilize to a limit cycle. The time series shown in Fig. 8(a) and its phase portrait projection, Fig. 8(b), use model parameters of $A^{*}=0.23$ and $\Gamma=3.75$ to achieve this. The transient period has been removed from both of the plots. This has a striking resemblance to that of Shilnikov cycles [30], where homoclinic orbits combine to a saddle focus.

The phase portrait projection shows an orbit spiral toward a fixed point saddle focus, but whereas one would normally expect the solution to stay at the node, as in a critically damped system, this solution is repelled away when it reaches the center. The repelled trajectory then proceeds to complete a number of oscillations before the solution repeats

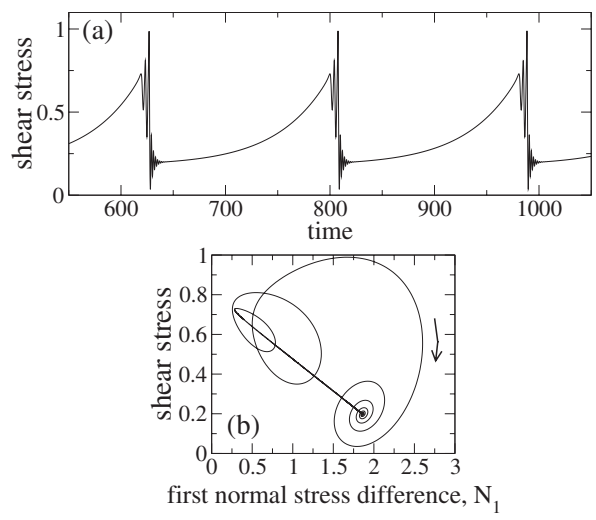

FIG. 8. Stable Shilnikov type limit cycle solutions that can be found in region $C$ of the phase diagram. $A^{*}=0.23$ and $\Gamma=3.75$. (a) Time series and (b) phase portrait shown. Arrow indicates direction of travel.
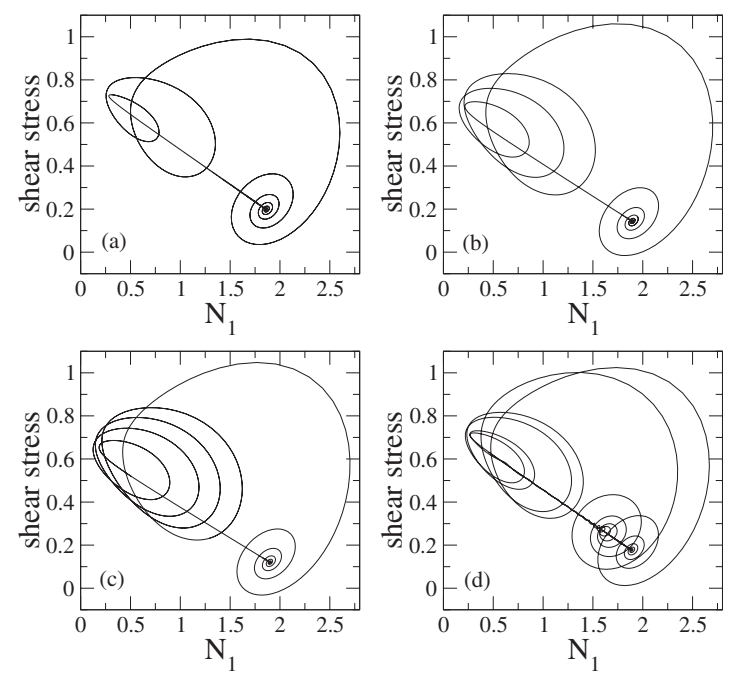

FIG. 9. Phase portrait projections of example solutions where the number of off spiral oscillations have increased from (a) the simplest observed with two oscillations to (b),(c) that of a greater number. In all solutions $A^{*}=0.23$ and from top left to bottom right $\Gamma=3.7, \Gamma=3.5, \Gamma=3.385$, and $\Gamma=3.6765$. The final plot (d) shows a period-2 bifurcation of the cycle. The plotted axis are the shear stress and the first normal stress difference $N_{1}$.

itself in the limit cycle. If one were to use the second normal stress difference $\left(N_{2}\right)$ as a third axis on the plot then the spiral would fall on a plane, and the ejected solution would be perpendicular. Shilnikov has proved theorems concerning stability and existence of such homoclinic periodic orbits $[30,31]$.

Similar Shilnikov orbits have been observed experimentally in systems such as optothermal nonlinear devices [32], liquid crystal flow [33], and even reverse spiral cycles in lasers with saturable absorbers [34].

The appearance of the stable solutions can also vary with the control parameter. A change is observed in the number of oscillations before returning to the spiral and also, in some cases, the number of spirals. As an example, several solutions where this change has been observed are shown in Fig. 9. These solutions are all calculated using the same value of $A^{*}=0.23$, but different values of $\Gamma$. Figures $9(\mathrm{a})-9(\mathrm{c})$ show an increase in the number of off spiral oscillations. Figure 9(d) shows a limit cycle with two spiral structures; this is a double-pulse homoclinic orbit version of Fig. 9(a).

We note that in comparison with the period-doubling chaos discussed above, this type of chaos has a much larger area in the parameter space $\Gamma-A^{*}$ and therefore it could be more easily observed in experiments. One would expect to see this type of behavior manifested as a type of bursting process in the stress during flow. Our results from the chaotic time series in Fig. 7 are similar to those seen in the chaotic flow of wormlike micelles [35].

\section{CONCLUSIONS AND OUTLOOK}

In conclusion, our generalized model for non-Newtonian flow investigated here contains a variety of nonlinear dy- 
namical phenomena arising from the shear induced forces of plane Couette flow. We have mapped the largest Lyapunov exponent of the solutions arising from varying the two main system parameters in order to identify the different dynamical regimes. Using this we were able to locate and observe clear bifurcation structures leading to chaotic solutions. We have also shown homoclinic Shilnikov saddle-focus orbits and revealed Shilnikov bifurcation. Through the use of stability analysis we were able to classify some of the main methods of stability loss that give rise to the chaotic solutions which are observed. Parametric bifurcation diagrams reveal the underlying structure of the equations, the boundaries of which match the Lyapunov exponent map very well. The mechanism causing smooth flow to become unstable is identified as Hopf bifurcation. We remark that the solution in Fig. 7 is similar to the stick-slip behavior seen in solid friction [36-39].

For spatially resolved flow, the full hydrodynamic problem must be considered, with the full model [Eq. (2)] includ- ing the Laplace-term and appropriate boundary conditions as suggested in [40]. Initial simulations are also seen to display chaotic responses analogous to inertial turbulence, but for very low Reynolds numbers $(R e<1)$. Such behavior could be utilized for potential mixing applications with complex fluids such as polymers, whereby the "self mixing" properties combine with the excellent phase space exploration that a chaotic system possesses.

Application to a cylindrical Couette flow where we expect to find shear banding in certain parameter regimes is possible and desirable. This then opens a wider possibility to compare with experiments $[41,42]$ and with other theoretical models $[43,44]$.

\section{ACKNOWLEDGMENTS}

C.G. and A.B. acknowledge the support of EPSRC U.K.
[1] O. Hess and S. Hess, Physica A 207, 517 (1994).

[2] O. Hess, C. Goddard, and S. Hess, Physica A 366, 31 (2006).

[3] P. Fischer, E. K. Wheeler, and G. G. Fuller, Physica A 41, 25 (2002).

[4] R. Landolt and P. Fischer, Proceedings of the 6th European Conference on Rheology, edited by H. Muenstedt, J. Kaschta and A. Merten, (Erlangen, Germany, 2002), pp. 559-560.

[5] M. Cates, D. Head, and A. Ajdari, Phys. Rev. E 66, 025202 (2002).

[6] A. Aradian and M. Cates, Europhys. Lett. 70, 397 (2005).

[7] D. Head, A. Ajdari, and M. Cates, Europhys. Lett. 57, 120 (2002).

[8] M. Das, B. Chakrabarti, C. Dasgupta, S. Ramaswamy, and A. Sood, Phys. Rev. E 71, 021707 (2005).

[9] S. Fielding and P. Olmsted, Phys. Rev. Lett. 92, 084502 (2004).

[10] J. Dasan, T. Ramamohan, A. Singh, and P. R. Nott, Phys. Rev. E 66, 021409 (2002)

[11] S. Hess, Z. Naturforsch. A 30, 728 (1975); 30, 1224 (1975).

[12] S. Hess, Z. Naturforsch. A 31, 1507 (1976).

[13] C. P. Borgmeyer and S. Hess, J. Non-Equil. Thermodyn. 20, 359 (1995).

[14] S. Hess, Z. Naturforsch. A 31, 1034 (1976).

[15] M. Doi, J. Polym. Sci., Polym. Phys. Ed. 19, 229 (1981).

[16] M. G. Forest, Q. Wang, and R. Zhou, Rheol. Acta 44, 80 (2004)

[17] G. Rienäcker and S. Hess, Physica A 267, 294 (1999).

[18] G. Rienäcker, M. Kröger, and S. Hess, Physica A 315, 537 (2002).

[19] S. Hess and M. Kröger, J. Phys.: Condens. Matter 16, S3835 (2004).

[20] R. Bird, R. Armstrong, and O. Hassager, Dynamics of Polymeric Liquids Vol. 1 and Vol. 2 (Wiley, New York, 1997).

[21] H. J. Wilson and S. M. Fielding, J. Non-Newtonian Fluid Mech. 138, 181 (2006).

[22] M. Johnson and D. Segalman, J. Non-Newtonian Fluid Mech. 2, 255 (1977).

[23] M. Grosso, S. Crescitelli, E. Somma, J. Vermant, P. Moldenaers, and P. Maffettone, Phys. Rev. Lett. 90, 098304 (2003).
[24] A. Wunenburger, A. Colin, J. Leng, A. Arneodo, and D. Roux, Phys. Rev. Lett. 86, 1374 (2001).

[25] H. Azzouzi, J. Decruppe, S. Lerouge, and O. Greffier, Eur. Phys. J. E 17, 507 (2005).

[26] J.-B. Salmon, A. Colin, and D. Roux, Phys. Rev. E 66, 031505 (2002).

[27] R. Bandyopadhyay and A. Sood, Europhys. Lett. 56, 447 (2001).

[28] A. Groisman and V. Steinberg, Nature (London) 405, 53 (2000).

[29] E. Doedel et al., Technical Report, Concordia University, 2002.

[30] L. P. Shilnikov, Sov. Math. Dokl. 6, 163 (1965).

[31] A. Shilnikov, L. Shilnikov, and D. Turaev, Moscow Math. J. 5, 269 (2005).

[32] R. Herrero, P. Pons, J. Farjas, F. Pi, and G. Orriols, Phys. Rev. E 53, 5627 (1996).

[33] T. Peacock and T. Mullin, J. Fluid Mech. 432, 369 (2001).

[34] D. Hennequin, F. Tomasi, B. Zambon, and E. Arimondo, Phys. Rev. A 37, 2243 (1988).

[35] R. Ganapathy and A. Sood, Phys. Rev. Lett. 96, 108301 (2006).

[36] H. Yoshizawa, Y.-L. Chen, and J. Israelachvili, Wear 168, 161 (1993).

[37] L. Bocquet, E. Charlaix, S. Ciliberto, and J. Crassous, Nature (London) 396, 735 (1998).

[38] B. Persson, Sliding Friction (Springer, New York, 2000).

[39] S. Hess, S. Heidenreich, P. Ilg, C. Goddard, and O. Hess, Atti Accad. Peloritana Pericolanti, Cl. Sci. Fis., Mat. Nat. LXXXVI, C1S0801010 (2008).

[40] S. Heidenreich, P. Ilg, and S. Hess, Phys. Rev. E 75, 066302 (2007).

[41] S. Lerouge, M. Argentina, and J. P. Decruppe, Phys. Rev. Lett. 96, 088301 (2006).

[42] Y. T. Hu and A. Lips, J. Rheol. 49, 1001 (2005).

[43] P. D. Olmsted, O. Radulescu, and C.-Y. D. Lu, J. Rheol. 44, 257 (2000).

[44] L.F. Rossi, G. McKinley, and L.P. Cook, J. Non-Newtonian Fluid Mech. 136, 79 (2006). 